Produto \& Produção, vol. 11, n. 3, p. 45-58, out. 2010

\title{
AHP MAKH-ER: Validação de um sistema de apoio à decisão para estudar a influência da pressão do tempo e da falta de informação no processo decisório
}

\author{
Kathiane Benedetti Corso \\ Escola de Administração - PPGA/EA/UFRGS \\ kathicorso@yahoo.com.br \\ Mauri Leodir Löbler \\ Programa de Pós-Graduação em Administração - PPGA/UFSM \\ lobler@ccsh.ufsm.br
}

\begin{abstract}
Este trabalho descreve a validação do Sistema de Apoio à Decisão $A H P M A K H$-ER, o qual possibilita estudar o comportamento dos decisores sob pressão do tempo e falta de informação. O AHP MAKH-ER foi estruturado com o Método Multicritério AHP - Processo Analítico Hierárquico, que por meio de uma seqüência de comparações aos pares das alternativas para determinados critérios, otimiza o processo de tomada de decisão, ranqueando as alternativas julgadas para a decisão final. Após validação, baseada na avaliação pelo usuário e na validação de mapeamento do processo, acredita-se que o AHP MAKH-ER pode ser utilizado como ferramenta de apoio para se estudar a pressão do tempo e a falta de informação no processo decisório, identificando diferentes comportamentos elencados pela literatura. Para pesquisas futuras, sugere-se que o AHP MAKH-ER seja aplicado, em forma de experimento, sob diferentes combinações das duas variáveis, aprofundando o entendimento sobre o comportamento do decisor.
\end{abstract}

Palavras-chave: Sistema de Apoio à Decisão, Pressão do Tempo, Falta de Informação

This paper describes the validation of the Decision Support System AHP MAKH-ER, which allows studying the decision makers' behavior under time pressure and missing information. AHP MAKH-ER was structured with Method Multicriteria AHP - Analytical Hierarchy Process, which through a sequence of comparisons to pairs of alternatives for certain criteria, optimizes the process of decision making, ranking the alternatives judged for the final decision. After validation, based on user-assessment and validation of process mapping, it is believed that the AHP MAKH-ER can be used as a support tool for studying the time pressure and missing information in decision making, identifying the different behaviors highlighted by the literature. For future research, suggests that the AHP MAKH-ER is applied in the form of experiment, under different combinations of two variables, deepening the understanding of decision maker's behavior.

Keywords: Decision Support System. Missing Information. Time Pressure

\section{Introdução}

O processo decisório, apesar de complexo, constitui-se em uma tarefa corriqueira do indivíduo, que a realiza consciente ou inconscientemente (KAHNEMAN; TVERSKY, 2000). Por se tratar de uma atividade não descartável e de fundamental importância, os estudos do processo decisório refletem a necessidade da busca permanente de aprimoramento da capacidade de decidir.

Nesse contexto, Fisher, Chengalur-Smith e Ballou (2003) afirmam que a eficácia da tomada de decisão é influenciada por diversos fatores, entre eles, o tempo disponível antes da decisão ser tomada. Hahn, Lawson e Lee (1992) afirmam que diante da pressão do tempo, a pessoa pode não ser capaz de manter o controle adequado sobre o processamento de todas as partes da informação, possibilitando como resultado, uma queda no desempenho.

Influente também no processo decisório é a disponibilidade da informação. Muitas das decisões cotidianas podem envolver situações em que haja a falta de informações. Jagacinski (1991) assegura que um problema bastante comum enfrentado diariamente ao tomar decisões e fazer avaliações é quando partes importantes da informação estão faltando. Kivetz e Simonson (2000) verificaram que a falta de informação afeta a preferência de escolha, e gostos em escolhas posteriores dos indivíduos. Ahituv, Igbaria e Sella (1998) corroboram 
nesse sentido, perante a falta de informação o desempenho da tarefa que o indivíduo está realizando fica comprometido.

Diante de diversos fatores que influenciam o processo decisório, estudos vêm sendo desenvolvidos a fim de se entender os processos de tomada de decisão e o comportamento dos indivíduos em presença de tais influências. Os Sistemas de Apoio a Decisão (SAD) além de serem sistemas que são utilizados como ferramenta estratégica nas empresas, são uma ferramenta de pesquisas acadêmicas que buscam estudar o comportamento dos decisores. Payne, Bettman e Johnson (1993) afirmam que um dos pontos chaves dos sistemas que apóiam decisões é a identificação da estratégia que está sendo empregada pelo indivíduo. Um exemplo é o Sistema Mouselab, criado por Johnson, Payne, Schkade e Bettman em 1991, que monitora o comportamento da aquisição e processamento da informação dos indivíduos, como: qual informação o sujeito procura, a seqüência da aquisição da informação, a quantidade de informação que ele adquiriu, e por quanto tempo a informação foi examinada.

No entanto, assim como qualquer instrumento de pesquisa, um Sistema de Apoio à Decisão também precisa ser validado para conferir credibilidade (GARDNER ET AL., 1993 APUD BIASIO ; BORENSTEIN, 2006). Quando a função de um SAD está relacionada com a utilização para pesquisas, o mesmo deve ser validado, com o intuito de ser instrumento útil para mensurar o que realmente se quer. Assim, segundo Hoppen, Lapointe e Moreau (1996), validação significa que a medida é o elo de ligação entre o problema que se quer investigar, ou a teoria que se quer confirmar, e a realidade que se quer observar. Apesar de poucos, no Brasil destacam-se os estudos recentes sobre validação de SAD de Biasio e Borenstein (2006) e Löbler e Hoppen (2006).

Nesse sentido, este trabalho tem por objetivo descrever a validação do SAD AHP MAKH-ER, sistema concebido pelos autores, que possibilita estudar o comportamento dos decisores sob pressão do tempo e falta de informação, estruturado com o Método Multicritério AHP - Processo Analítico Hierárquico. Este método, propõe que por meio de uma sequiência de comparações aos pares das alternativas para determinados critérios, é possível otimizar o processo de tomada de decisão, ranqueando as alternativas julgadas para a decisão final (SAATY, 1991).

O artigo apresenta inicialmente o referencial teórico sobre Validação de SAD e sobre o Método Multicritério AHP. Em seguida é apresentado o AHP MAKH-ER, seu funcionamento original e no contexto da pressão do tempo e da falta de informação, bem como a validação pelo usuário do sistema, e a validação do mapeamento do processo decisório. Por fim, são feitas as considerações finais.

\section{Conceitos}

\subsection{Sistemas de apoio à decisão e sua validação}

Um Sistema de Apoio à Decisão (SAD) é um sistema computadorizado, interativo, que proporciona ao tomador de decisão modelos e dados para apoiar tarefas de decisão estruturadas ou não-estruturadas (PAYNE; BETTMAN; JOHNSON, 1993). Contudo, Biasio e Borenstein (2006) citam Gardner et al. (1993) os quais asseveram que independentemente dos objetivos e contexto de sua utilização, um SAD só pode ser utilizado com confiança se devidamente validado.

Borenstein (1998) lembra que é necessário fazer a distinção entre os conceitos de validação e avaliação. Segundo o autor, a validação é o processo de definir se o comportamento do modelo representa o sistema do mundo real num problema específico. Já a avaliação é o processo de avaliar o software como um todo, tanto os seus comportamentos internos (ausência de erros de funcionamento) como no que se refere à utilidade para os usuários. A validação de um SAD não está preocupada em provar que o mesmo é uma representação verdadeira do mundo real, visto que isso é impossível, mas em demonstrar que um SAD possui relacionamentos subjacentes apropriados para permitir uma representação aceitável (BORENSTEIN; BECKER, 2000). Sendo assim, entende-se que a validação é parte do processo de avaliação de um SAD.

Apesar de Borenstein (1997, p. 67) já naquela época afirmar que "vários Sistemas de Apoio à Decisão (SAD) foram construídos para, interativamente, facilitar decisores na resolução de problemas envolvendo múltiplos critérios", a literatura sobre validação de SAD é bastante escassa, possivelmente refletindo a dificuldade dessa tarefa (BIASIO; BORENSTEIN, 2006). Nesse sentido, os autores asseguram que a validação de um SAD é considerada, ao mesmo tempo, uma etapa fundamental para tornar os sistemas informatizados mais eficientes e científicos, e uma tarefa extremamente difícil porque lida com a modelagem, análise e valoração do processo de tomada de decisões.

Neste estudo, para fins de validação, os principais elementos considerados são o uso e a utilidade do sistema, a avaliação da interface, a facilidade e compreensão do sistema e a satisfação do usuário. Logo, a validação é baseada na avaliação do sistema, em uma situação de tomada de decisão, em que uma tarefa é realizada pelos usuários. Desse modo, a fim de verificar a ausência de erros e, principalmente, se o AHP MAKH-ER representa os processos de decisão encontrados no mundo real, são testadas as funcionalidades do sistema. Outra forma de validação é baseada na avaliação feita pelos pesquisa- 
dores sobre o mapeamento do processo decisório, por meio da verificação do funcionamento do registro dos acessos computacionais quando do uso do sistema, e das análises relativas ao processo decisório.

\subsection{Método multicritério AHP: sustentando o processo decisório}

O Método Multicritério AHP - Processo Analítico Hierárquico - derivado do inglês Analytical Hierarchy Process - doravante neste artigo denominado Método AHP, é um método multi-objetivo de apoio à decisão criado por Saaty na década de 70 nos Estados Unidos (SAATY, 1991). Este método foi um dos primeiros a ser desenvolvido no ambiente das decisões multicritério, sendo hoje talvez o mais utilizado no mundo (GOMES; ARAYA ; CARIGNANO, 2004). No entender de Rafaeli e Müller (2007, s.p):

[...] o AHP surge como um método estruturado para a análise de problemas nos quais existem diversas variáveis a serem avaliadas simultaneamente em um contexto que envolve vários níveis e critérios, propiciando a investigação de critérios subjetivos de modo quantitativo. Este é um método que vem sendo amplamente discutido e as recentes inovações teóricas na área de modelos de tomada de decisão vêm reforçando a sua utilidade em diversas áreas do conhecimento.

O Método AHP permite estruturar uma decisão em níveis hierárquicos, determinando por meio da síntese de valores dos decisores, uma medida global para cada uma das alternativas, priorizando-as ou classificando-as ao final do método. Assim, este método permite medir todos os fatores importantes envolvidos num processo decisório (SAATY, 1991). O método possui quatro princípios fundamentais: (1) atributos e propriedades, onde um conjunto de alternativas é comparado em relação a um conjunto de propriedades; (2) correlação binária, quando dois elementos são comparados, baseados em uma determinada propriedade, realiza-se uma comparação binária, na qual um elemento pode ser preferível ou indiferente a outro; (3) escala fundamental, onde cada elemento associa-se a um valor de prioridade sobre os outros elementos, conforme escala de números positivos e reais; e, (4) hierarquia, em que os elementos são organizados por ordem de preferência e de forma homogênea em seus respectivos níveis hierárquicos.

A utilização de estudos e tarefas estruturadas com o Método AHP pode ser encontrada em diversos trabalhos sob diferentes contextos, como no desenvolvimento de software (SANGLARD JUNIOR e GOMES, 2003); na justificativa de investimentos em TI (BETENCOURT ; BORENSTEIN, 2002); na contabilidade e custeio (MATOS ; MOURA, 2002; GALVÃO; COGAN ; SANTOS, 2002; FREZATTI, et al., 2005); em logística (IAÑEZ; CUNHA, 2006); em estudos de conseqüências ambien- tais (JIMÉNEZ; MATEOS ; RÍOS-INSUA, 2009); na assistência médica (LIBERATORE ; NYDICK, 2008), dentre outros. Shimizu (2006) corrobora que o Método AHP tem sido empregado para situações de alocação de recursos, medida de desempenho, pesquisa de mercado, decisões estratégicas, previsão de cenários, resolução de conflitos, decisão sobre risco, bem como previsões políticas e sociais.

Por ser um método analítico hierárquico, o Método AHP hierarquiza em níveis os elementos da decisão a serem comparados. Conforme Saaty (1991) esta etapa é similar à construção de uma árvore de decisão (ex.: compra de carro na Figura 1): no topo, situa-se o objetivo maior (geral) e, logo abaixo, localizam-se os critérios associados ao problema de decisão. Estes, por sua vez, devem ser 'quebrados' mais detalhadamente em vários outros níveis (subcritérios), de acordo com a complexidade do problema. Finalmente, as alternativas são adicionadas na hierarquia, abaixo do último nível de critérios.

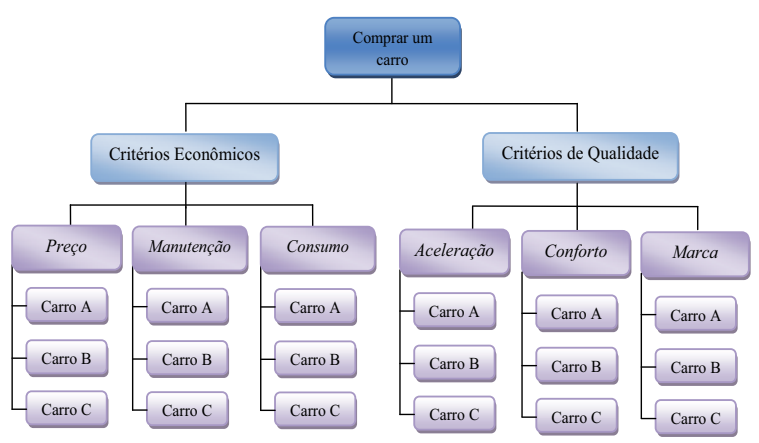

Figura 1 - Estrutura hierárquica para o problema de decisão Fonte: elaborado pelos autores adaptado de GOMES; ARAYA; CARIGNANO (2004)

Depois de construída a hierarquia da decisão, cada decisor deve fazer uma comparação, par a par, de cada elemento em um nível hierárquico dado, criando-se uma matriz quadrada de decisão. Saaty (1991) recomenda que o processo de comparar aos pares deva ser implementado utilizando perguntas verbais, em que o decisor deverá julgar a importância de um elemento sobre outro, a partir de uma escala linear própria que varia de 1 a 9 , denominada Escala Fundamental de Saaty, demonstrada no Quadro 1:

\begin{tabular}{|c|c|c|}
\hline Escala & $\begin{array}{c}\text { Intensidade da } \\
\text { Importância }\end{array}$ & Descriçao \\
\hline $\mathbf{1}$ & Mesma importância & $\begin{array}{c}\text { As duas atividades contribuem } \\
\text { igualmente para o objetivo. }\end{array}$ \\
\hline $\mathbf{3}$ & $\begin{array}{c}\text { Um pouco mais } \\
\text { importante }\end{array}$ & $\begin{array}{c}\text { A experiência e o juízo } \\
\text { favorecem uma atividade em } \\
\text { relação à outra. }\end{array}$ \\
\hline $\mathbf{5}$ & $\begin{array}{c}\text { Consideravelmente } \\
\text { mais importante }\end{array}$ & $\begin{array}{c}\text { A experiência ou juízo favorece } \\
\text { fortemente uma atividade em } \\
\text { relação à outra. }\end{array}$ \\
\hline
\end{tabular}




\begin{tabular}{|c|c|c|}
\hline $\mathbf{7}$ & $\begin{array}{c}\text { Muito mais impor- } \\
\text { tante }\end{array}$ & $\begin{array}{c}\text { Uma atividade é muito forte- } \\
\text { mente favorecida em relação à } \\
\text { outra. }\end{array}$ \\
\hline $\mathbf{9}$ & $\begin{array}{c}\text { Extremamente mais } \\
\text { importante }\end{array}$ & $\begin{array}{c}\text { A evidência favorece uma ativi- } \\
\text { dade em relação à outra, com o } \\
\text { mais alto grau de segurança. }\end{array}$ \\
\hline
\end{tabular}

Quadro 1 - Escala Fundamental de Saaty

Fonte: Saaty (1991), adaptado pelos autores

Após a criação da matriz quadrada recíproca positiva conhecida como matriz dominante, que expressa o número de vezes em que uma alternativa domina ou é dominada pelas demais (SAATY, 1991), o Método AHP gera um índice de consistência, que foi projetado para alertar o agente de decisão sobre as possíveis inconsistências nas comparações feitas. Exemplificando, suponha que nas respostas do agente de decisão o atributo A seja duas vezes mais importante que o $\mathrm{B}$, enquanto que o B seja três vezes mais importante que o C. Para ser corretamente consistente, o decisor deve julgar que o atributo A seja seis vezes mais importante que o atributo $\mathrm{C}$.

Dessa forma, a cada matriz de comparação, é calculado um auto-vetor (V1), que representa a importância relativa entre os elementos da matriz, e cujo somatório deve ser igual a 1. Para verificar se os dados obtidos estão logicamente relacionados, estima-se inicialmente o autovalor (V2), resultado da multiplicação do auto-vetor (V1) pela soma das colunas da matriz de comparação. A Razão de Consistência (RC) é calculada através da equação:

$\mathrm{RC}=\mathrm{IC} / \mathrm{IR} \quad$ onde $\quad \mathrm{IC}=\left(\mathrm{V}_{2}-\mathrm{n}\right) /(\mathrm{n}-1)$

Por meio desta notação, IC corresponde ao Índice de Consistência calculado pela fórmula apresentada, e IR é o Índice Randômico; um índice aleatório calculado para matrizes quadradas de ordem n pelo Laboratório Nacional de Oak Ridge (EUA). Saaty (1991) propõe uma tabela com os índices randômicos de matrizes de ordem n, visualizada no Quadro 2:

\begin{tabular}{|c|c|c|c|c|c|c|c|c|}
\hline $\mathbf{n}$ & $\mathbf{1}$ & $\mathbf{2}$ & $\mathbf{3}$ & $\mathbf{4}$ & $\mathbf{5}$ & $\mathbf{6}$ & $\mathbf{7}$ & $\mathbf{8}$ \\
\hline $\mathbf{I R}$ & 0.00 & 0.00 & 0.58 & 0.90 & 1.12 & 1.24 & 1.32 & 1.41 \\
\hline
\end{tabular}

Quadro 2 - Índice Randômico Médio do AHP para matrizes quadradas de ordem $n$

Fonte: Saaty (1991) adaptado pelos autores

Depois de calculado, quanto maior for o RC, maior será a inconsistência (GOMES; ARAYA; CARIGNANO, 2004). Deste modo, dependendo do tamanho da matriz (n), se dá a consistência ou não. Quando $n=2, \mathrm{RC}$ é nulo; $\mathrm{n}=3$, RC deve ser menor que 0,$05 ; \mathrm{n}=4$, RC deve ser menor que 0,$09 ; n>4$, uma inconsistência considerada aceitável é $\mathrm{RC} \leq 0,10$. A escolha da decisão final deverá basear-se naquela alternativa que obtenha o maior escore (peso percentual). Este cálculo é efetuado pela multiplicação dos pesos de cada critério relacionado à alternativa indicada. Ainda, o resultado final permite definir uma seqüência cardinal da importância dos atributos e das alternativas.

\section{Metodologia proposta}

\subsection{Método científico usado}

Este artigo é estruturado dentro de uma visão epistemológica positivista, o que segundo Hoppen, Lapointe e Moreau (1996) significa que o pesquisador entende que os fenômenos sociais são reais e podem ser estudados objetivamente. Segundo Burrel e Morgan (1979), os estudos do tipo positivista procuram explicar e predizer o que acontece no mundo social, através de uma procura de fenômenos regulares e de relações causais entre os elementos que o constituem, utilizando instrumentos de medida estruturados.

Quanto à abordagem qualitativa ou quantitativa, Simonetto e Borenstein (2008), afirmam que, basicamente, existem duas técnicas que podem ser utilizadas na prática de validações e, consiste de: validação qualitativa, que utiliza comparações subjetivas de desempenho, sendo: validação de face, validação preditiva, testes de campo, análise de sensibilidade e interação visual. A validação Quantitativa utiliza técnicas estatísticas para comparar o desempenho do SAD com casos práticos de teste. De acordo com a classificação anterior, neste trabalho a validação é classificada como qualitativa.

Para o atendimento dos objetivos descritos neste trabalho, foi utilizado o procedimento de análise consolidada pela Pesquisa Operacional. Segundo o trabalho de Biasio (2002), um estudo de Pesquisa Operacional consiste, basicamente, em construir um modelo de um sistema real existente como meio de analisar e compreender o comportamento dessa situação, com o objetivo de levá-lo a apresentar o desempenho que se deseja. A pesquisa realizada seguiu as fases usualmente utilizadas em um estudo de Pesquisa Operacional: (1) percepção ou demanda por solução; (2) definição do problema; (3) construção do modelo; (4) solução do modelo; (5) validação do modelo. A última fase, implementação dos resultados, não foi operacionalizada, pois justamente trata-se da validação do sistema.

Tomando como base, e adaptando o método proposto pela Pesquisa Operacional, as etapas da construção desse artigo, que culmina com a validação do sistema, são divididas em quatro fases:

- Etapa 1 - Identificação de Necessidade ou Problema: após revisão de literatura sobre o tema, verificou-se a necessidade da construção de um Sistema de Apoio à Decisão que pudesse ser utilizado para estudar o 
comportamento de decisores sob pressão do tempo e falta de informação.

- Etapa 2 - Definição da Tarefa e dos Multicritérios: definiu-se como tarefa experimental a escolha de um automóvel, e posteriormente seus respectivos critérios e alternativas. Nesta mesma etapa foi feito um estudo de como embutir as variáveis pressão do tempo e falta de informação na tarefa a ser executada.

- Etapa 3 - Construção da Ferramenta: o SAD foi construído com base no Método Multicritério AHP - Processo Analítico Hierárquico. Inicialmente, um módulo chamado Gerenciador foi arquitetado, o qual permite ao pesquisador elaborar a tarefa e manipular as variáveis, critérios e alternativas a serem utilizadas na mesma. Este Gerenciador possibilita a criação do módulo Tarefa, que é aquele a ser executado pelo sujeito experimental.

- Etapa 4 - Validação e Análise dos Resultados: A validação do sistema construído se deu por meio de duas validações, a do usuário e a de mapeamento. A validação pelo usuário do SAD buscou avaliar a ferramenta quanto à sua utilidade, interface, facilidade e satisfação. Já a validação de mapeamento do processo visou mapear os $\log s$ do sistema por meio do registro automático e da catalogação das ações tomadas pelo decisor para chegar a sua escolha final. Os resultados foram analisados e permitiram validar o Sistema.

\section{Ferramenta proposta}

\subsection{AHP MAKH-ER: funcionamento, contexto e validação}

Visando construir um SAD em que pudesse se estudar a pressão do tempo e a falta de informação no processo decisório, foi desenvolvido o software AHP $M A K H-E R$, sustentado pela metodologia multicritério AHP. Nessa seção é apresentado o funcionamento do software, demonstrando a estrutura geral e seu modo de operação no contexto da pressão do tempo e da falta de informação, e por fim, a validação do mesmo.

\subsubsection{Funcionamento do AHP MAKH-ER}

$O$ software AHP MAKH-ER foi desenvolvido em dois módulos: o AHP MAKH-ER - Gerenciador, destinado ao pesquisador, que possibilita criar qualquer tipo de tarefa decisória, e que gera então o AHP MAKH-ER - Tarefa, destinado ao tomador de decisão. Por meio do modelo matemático subjacente, o Método AHP, o sistema apóia os indivíduos na tarefa de decisão e auxilia o pesquisador no mapeamento do processo decisório, através dos acessos computacionais $(\log s)$, ou seja, da gravação dos movimentos e acessos realizados pelos decisores. Desenvolvido com a ferramenta Windev XI para ser executado em ambientes Microsoft Windows ou Linux, o AHP MAKH-ER não precisa necessariamente ser instalado no computador, bastando executar diretamente os arquivos AHP_Gerenciador.exe e AHP_Tarefa.exe para ser iniciado. Após a execução da tarefa são gerados pelo sistema, arquivos que servem de informação para o pesquisador; sendo (1) log.xls, e (2) resultado.xml.

\subsubsection{A tarefa e a construção das alternativas e atributos}

A fim de ilustrar como o AHP MAKH-ER é estruturado e foi validado, apresenta-se uma tarefa decisória que consiste na escolha de um automóvel popular. A tarefa possui como alternativas automóveis populares e como atributos, suas características. Como alternativas da tarefa foram escolhidos três carros populares fabricados e disponíveis no Brasil, todos com seus critérios atualizados no segundo semestre de 2008. A escolha pelos modelos Gol, Palio e Celta deu-se com base na lista dos 50 modelos mais vendidos no mercado nacional no primeiro semestre de 2008, segundo dados da Federação Nacional da Distribuição de Veículos Automotores (Fenabrave) encontrados no site da Revista Quatro Rodas. Optou-se então por escolher o mais básico de cada modelo encontrado: Gol City, Palio Fire e Celta Life, todos modelo hatch, motor 1.0, quatro portas, bicombustível e ano 2008 .

Para a definição dos critérios foram empregados os seis critérios mais relevantes para escolha de um carro, utilizados no estudo de Löbler (2005): preço do carro, economia de combustível, conforto, desempenho do carro, custo de manutenção e marca. Estes critérios foram definidos por Löbler (2005) com base em revistas especializadas, contatos com indivíduos que possuem conhecimento no ramo de automóveis, e principalmente, a partir de uma pesquisa realizada pela IBOPE Solution em 2002, encomendada pela União da Agroindústria Canavieira de São Paulo. Apenas o critério desempenho foi renomeado, pois se entendeu que ficaria melhor representado por tempo de aceleração. Os seis critérios ficaram denominados na tarefa da seguinte forma: preço, consumo, conforto, marca, aceleração e manutenção. A partir de consultas à Revista Quatro Rodas - on-line, bem como busca com especialista do ramo, obtiveramse as informações dos critérios utilizados.

\subsubsection{Interface e funcionamento do sistema de apoio à decisão AHP MAKH-ER}

Após ser gerada através do AHP MAKH-ER - Gerenciador, a tarefa decisória está pronta para ser aplicada. A tela inicial da tarefa experimental visualizada pelo decisor é apresentada na Figura 2. O AHP MAKH-ER Tarefa oferece nas linhas as três alternativas de escolha, 
isto é, os três modelos de automóveis disponíveis para a escolha dos decisores (Gol City, Palio Fire e Celta Life).

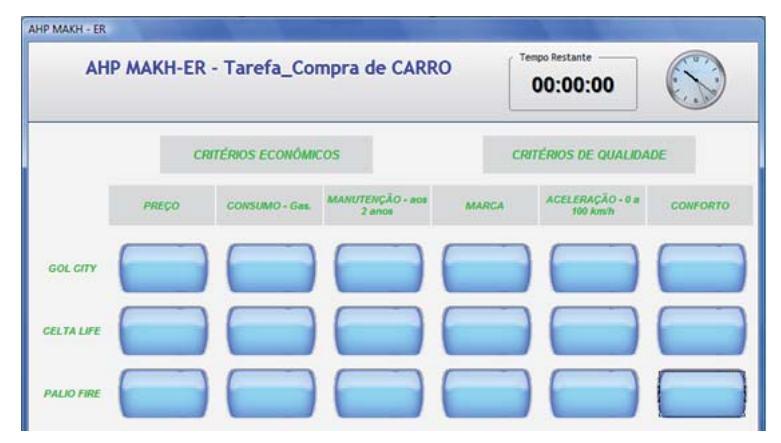

Figura 2 - Tela inicial da tarefa do AHP MAKH-ER - Tarefa Fonte: Sistema desenvolvido para a pesquisa

Nas colunas encontram-se os atributos de cada automóvel (Preço, Manutenção, Consumo, Aceleração, Conforto e Marca), ou seja, os critérios relevantes na compra de carros. A ordem em que aparecem as alternativas e os critérios foi definida por meio de sorteio, a fim de evitar contaminação em função da preferência dos pesquisadores. Ao abrir a tela, os sujeitos se deparam com todas as células azuis que contém a informação da alternativa X critério, fechadas; isto é, as informações não estarão disponíveis inicialmente, pois dessa forma pode-se verificar posteriormente como o decisor navega no sistema. Para verificá-las, é necessário um click sobre cada célula, sem ordem estabelecida, ficando a critério do decisor quais as informações e a ordem em que ele quer examinar.

Sendo o AHP um método estruturado em níveis, além das alternativas e critérios, os decisores encontram os Grupos de Critérios, assim definidos neste estudo. Logo, para a decisão de "comprar um carro", os seis critérios de escolha são agrupados em um nível hierarquicamente superior, divididos e nomeados aqui em Critérios Econômicos e Critérios de Qualidade, conforme validado por três especialistas no assunto.

As escolhas e julgamentos iniciam no momento em que o decisor examina todos os valores das alternativas de um critério qualquer, sendo aberta uma tela automática, do tipo pop up (Figura 3), com uma pergunta indagando o decisor a fazer uma comparação, par a par, de cada elemento, conforme metodologia do Método AHP.

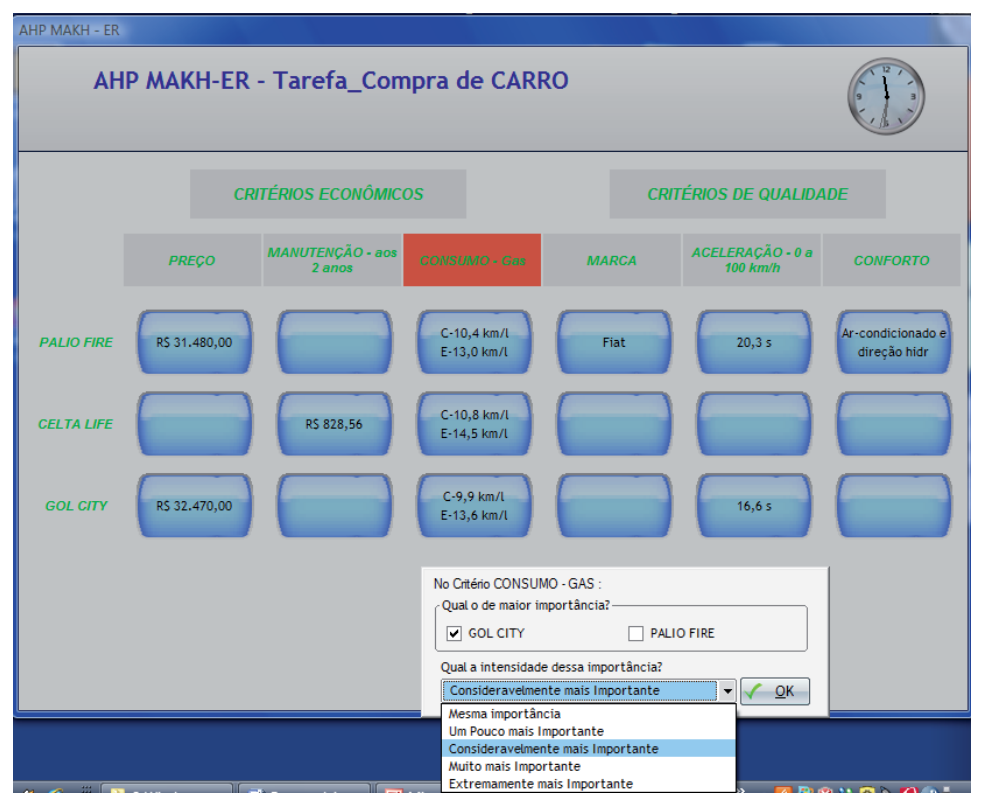

Figura 3 - Tela da tarefa do AHP MAKH-ER - Tarefa com abertura da tela de julgamento no primeiro nível Fonte: Sistema desenvolvido para a pesquisa

De tal modo, o decisor representará, a partir da Escala Fundamental de Saaty, sua preferência entre os elementos comparados. Seguindo a recomendação de Saaty (1991) para que o processo de comparação aos pares seja por meio de perguntas verbais, no caso da Figura 3 o decisor terá que comparar entre: Consumo/ Gol City x Consumo/Palio Fire, Consumo/Palio Fire x Consumo/Celta Life, e Consumo/Gol City x Consumo/ Celta Life, sempre dois a dois, respondendo a pergunta: "No Critério Consumo, qual o de maior importância?", ou seja, julgará qual dos dois contribui mais para a maximização do critério, conforme sua opinião. Em seguida, é questionado: "Qual a intensidade dessa importância?", isto é, quantas vezes o Consumo do Celta Life é melhor que o Consumo do Gol City, conforme exemplo.

O mesmo processo, da tela automática do tipo pop up, se dará para os demais critérios sempre que o decisor abrir todas as células das alternativas de um critério. 
Se, para um determinado critério, examinar apenas as alternativas dos carros Gol City e Celta Life, por exemplo, a tela pop up das comparações não abrirá, sendo necessária a abertura das três alternativas (Gol City, Palio Fire e Celta Life). Depois de ter passado pelo procedimento demonstrado na Figura 3, para todos os critérios de um Grupo de Critérios, é aberta uma nova tela pop up de comparação, para um nível hierárquico superior. Após o decisor ter verificado as alternativas dos critérios Preço, Manutenção, e Consumo, ambos pertencentes ao Grupo de Critérios Econômicos, uma tela de comparação entre esses três critérios será apresentada para o decisor comparar (Preço X Consumo; Preço X Manutenção; e Manutenção X Consumo) e hierarquizar, isto é: "No grupo de Critérios Econômicos, qual o de maior importância?", seguindo os mesmos passos das comparações do primeiro nível (Figura 3).

O mesmo se dará para os critérios do Grupo de Critérios de Qualidade, desde que as alternativas dos mesmos já tenham sido visualizadas pelo indivíduo. Após ter examinado os seis critérios, se parte para o nível hierárquico superior, para a comparação entre os Critérios Econômicos e os Critérios de Qualidade (Critérios Econômicos X Critérios de Qualidade), com a tela automática questionando: "Entre os grupos de Critérios, qual o de maior importância?".

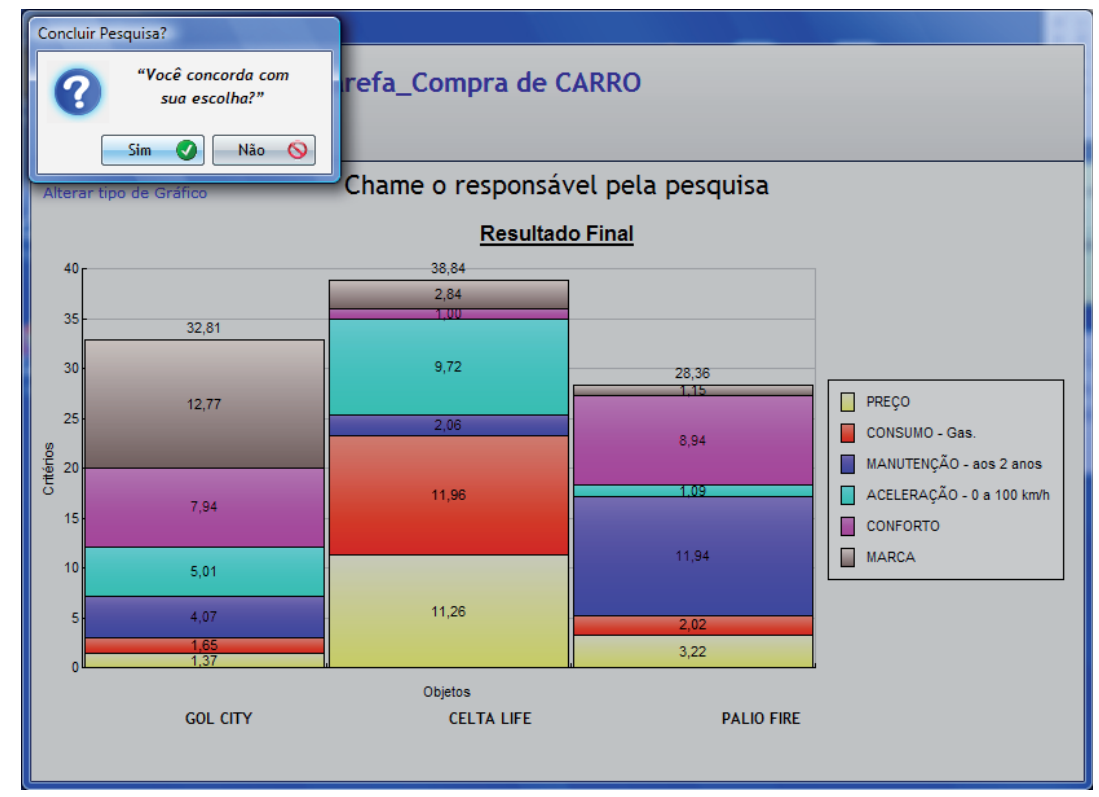

Figura 4 - Tela final do AHP MAKH-ER - Tarefa com gráfico da escolha Fonte: Sistema desenvolvido para a pesquisa

Por fim, os pesos são obtidos e a consistência da matriz é verificada, processo feito automaticamente pelo $A H P$ $M A K H-E R$. Os pesos são utilizados para verificar a importância relativa de cada critério. Este processo é denominado de normalização da matriz, devendo ser seguido pela determinação do índice de consistência da matriz, a fim de garantir os resultados das comparações paritárias (SAATY, 1991). A escolha da decisão final deverá basear-se naquela alternativa que obtenha o maior escore (peso percentual). Este cálculo é efetuado pela multiplicação dos pesos de cada critério relacionado à alternativa indicada. Como tela final da decisão de cada usuário, é apresentada em forma de gráfico de barras verticais (Figura 4), a escolha do automóvel, conforme alternativa que obtenha o maior escore (peso percentual), com as referidas porcentagens de cada critério que ele julgou ser importante durante a tarefa.

No gráfico, o decisor poderá visualizar os pesos de cada critério, ou seja, além de verificar qual a alternativa da sua preferência, ele visualiza quais os critérios que estão com maior e menor influência na escolha, conforme sua ponderação dada para cada critério em cada modelo de carro. Na mesma tela é indagado ao decisor se ele concorda com a escolha. Se não concordar com a alternativa escolhida, ele pode optar por clicar em "não", que o sistema direcionará para a tela inicial, cabendo a ele modificar e reponderar aquele(s) critério(s) no(s) qual(ais) não tenha concordado. Caso concorde com a escolha final, clicando em "sim", o sistema será finalizado.

\subsection{O AHP MAKH-ER no contexto da pressão do tempo e da falta de informação}

Com intuito de desenvolver um SAD que permita estudar a pressão do tempo e a falta de informações, o AHP MAKH-ER possibilita que se façam algumas interferências a fim de se estudar tais variáveis. 


\subsubsection{Aplicando a pressão do tempo}

A fim de estudar o comportamento dos indivíduos sob pressão do tempo, podendo ser gerado um cenário sob essa variável, fez-se o resgate na literatura. Destacamse os conceitos de Smith e Hayne (1997), de que a pressão de tempo é vivenciada através do uso de limites de tempo impostos para a execução de uma tarefa; de Fisher, Chengalur-Smith e Ballou (2003) que asseguram que a pressão do tempo é experienciada sempre que o tempo viável para a conclusão de uma tarefa é percebido como sendo mais curto do que normalmente exigido para a atividade; bem como de Ordóñez e Benson (1997) que indicam que essa restrição de tempo induz algum sentimento de estresse e cria uma necessidade de lidar com o tempo limitado.

Para criar uma situação de pressão do tempo ao decisor, no módulo AHP MAKH-ER - Gerenciador, na aba "Configuração de Tempo" (Figura 5), o pesquisador pode habilitar um contador de tempo, escolhendo o tempo em que a tela da tarefa ficará disponível para o decisor. Após o período definido, automaticamente a tarefa é abortada, e o sistema indica que o tempo para responder a pesquisa acabou, tolerando apenas 5 segundos até a tela ser fechada.

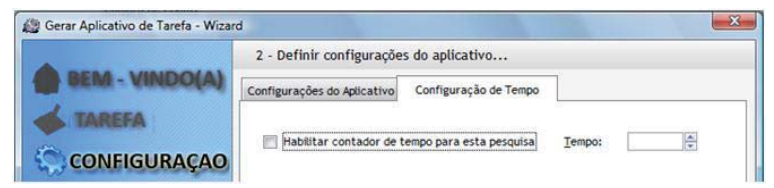

Figura 5 - Aba "Configuração de Tempo" da Janela Configuração do Gerador de Aplicativo de Tarefa assistente do AHP MAKH-ER - Gerenciador Fonte: Sistema desenvolvido para a pesquisa

Com base nesses conceitos, além de se estipular um determinado tempo para a realização da tarefa, gerando o cenário da pressão do tempo, sugere-se que o tempo seja cronometrado, e relembrado a cada momento pré-estabelecido, como forma de exercer pressão ao indivíduo. Caso o tempo termine, e o decisor não tenha examinado todas as informações, e realizado todas as comparações, o sistema preencherá com 1 (mesma importância) as matrizes faltantes, inferindo que, como o indivíduo não as examinou, tenha então o mesmo peso. O que diferenciará na escolha final, serão aquelas alternativas que foram analisadas e hierarquizadas pela escala, e que serão apresentadas no gráfico.

Dentre várias implicações da pressão do tempo no processo decisório, Payne, Bettman e Luce (1996) destacam que quando sob severa pressão do tempo as pessoas aceleram o processamento da informação, se tornam mais seletivas, e mudam as estratégias de um padrão de processamento mais profundo, baseado em alternativas, para um mais amplo, baseado em atributos (critérios).

\subsubsection{Aplicando a falta de informação}

Para o estudo do comportamento do decisor na realização de uma tarefa com falta de informação, no módulo AHP MAKH-ER - Gerenciador, é possível manipular a disponibilidade de informação da tarefa, ou seja, a variável falta de informação. Para definir uma tarefa com falta de informação, foi considerada aquela em que alguns valores de determinadas células de alternativa $X$ critério foram retirados. Isto porque a literatura afirma que a falta de informação se dá quando nem todas as informações são disponibilizadas ao indivíduo (KORNER et al., 2007). Como pode ser visto na Figura 6, na aba "Exibir Valores", o pesquisador escolhe quantas e para quais alternativas X critérios ocultará a informação. No exemplo, foram ocultados aleatoriamente o preço do Celta Life, o consumo do Gol City, a aceleração do

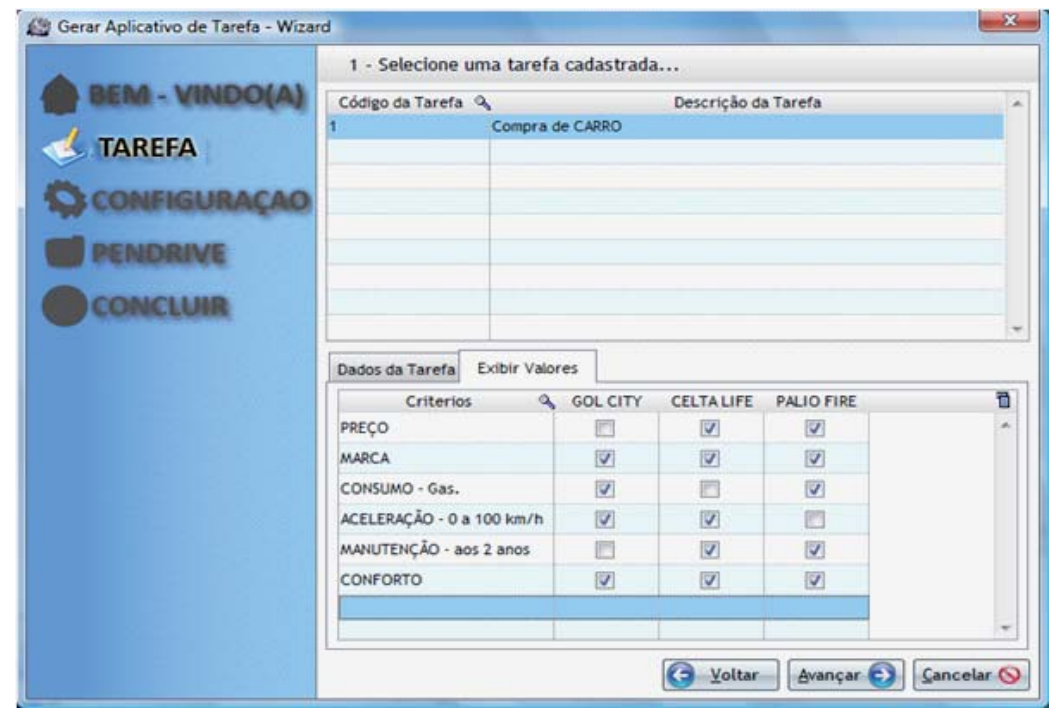

Figura 6 - Aba "Exibir Valores" da Janela Tarefa do Gerador de Aplicativo assistente do AHP MAKH-ER - Gerenciador Fonte: Sistema desenvolvido para a pesquisa 
Palio Fire, o conforto do Gol City, a manutenção do Celta Life, e a marca do Palio Fire.

A Figura 7 apresenta a interface da tarefa decisória do AHP MAKH-ER - Tarefa com informação faltante, em que são retirados alguns valores de determinados critérios dos automóveis, por meio de sorteio. Ao clicar em uma célula previamente estabelecida para não disponibilizar a informação, o decisor irá se deparar com a frase "Sem Valor" naquela célula. Mesmo assim, ele terá de prosseguir com o julgamento, tendo que escolher entre as duas alternativas apresentadas.

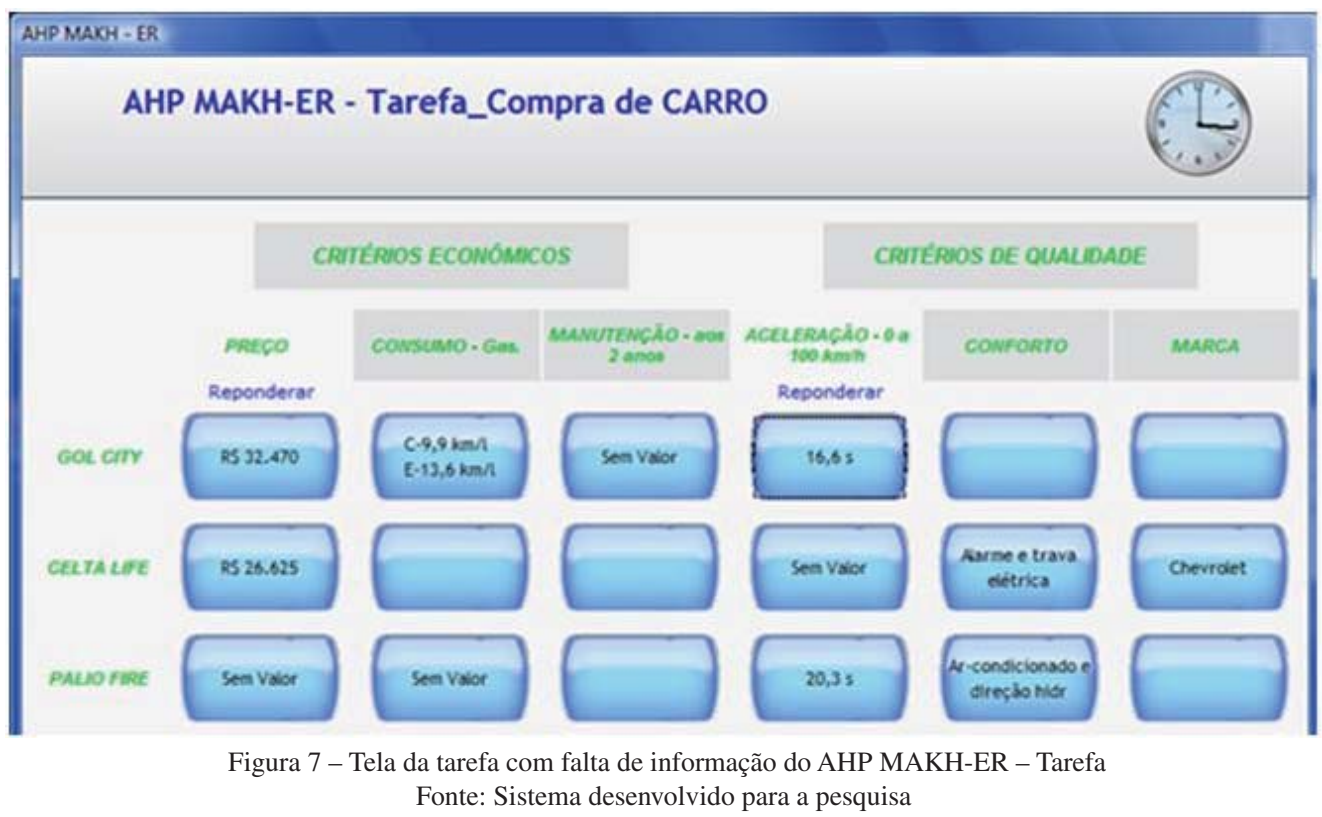

Segundo Jagacinski (1991) diversas são as constatações sobre como os decisores agem quando estão diante de uma tarefa com informação faltante. $\mathrm{O}$ autor ressalta que estudos relatam que os tomadores de decisão ignoram a falta de informação, ou lançam a hipótese de que os decisores assumem um valor constante para a dimensão da informação (geralmente uma média ou um pouco abaixo). Outros sugerem que o tomador de decisão prediz a informação faltante com base na informação disponível. O objetivo do AHP MAKH-ER é permitir o mapeamento do processo decisório, sob a falta de informação, possibilitando a verificação dos diferentes comportamentos do decisor.

\section{Validação e análise dos resultados}

\subsection{Validação pelo usuário do sistema de apoio à decisão AHP MAKH-ER}

Para testar e então validar o AHP MAKH-ER, vinte e dois tomadores de decisão, alunos de graduação, participaram da validação. De início, foi feita uma breve introdução sobre o Método AHP adotado no sistema, apresentando a tela inicial do mesmo. A apresentação foi seguida por uma leitura das instruções aos usuários do AHP MAKH-ER, que acompanharam individualmente com uma cópia impressa dessas instruções. Ressalta-se que para a validação do software, as variáveis pressão do tempo e falta de informação não foram embutidas na tarefa decisória, focando no objetivo inicial de validar o sistema.

Após a utilização do sistema e a visualização da decisão final (gravadas em arquivo digital), um questionário adaptado de Löbler (2005) foi aplicado com os usuários a fim de se avaliar o sistema AHP MAKH-ER e seu uso. São apresentadas a seguir a avaliação do sistema $A H P$ $M A K H-E R$, obtida a partir das informações fornecidas pelos decisores no questionário aplicado, que tem a finalidade de verificar a percepção dos mesmos. Os resultados são apresentados por item avaliado, numa escala de 1 (concordo plenamente) a 5 (discordo plenamente), de forma quantitativa; e qualitativamente a partir das observações e sugestões fornecidas pelos usuários na questão aberta.

Na Tabela 1 observa-se a avaliação do usuário quanto aos itens considerados: o uso e a utilidade do sistema, a avaliação da interface, a facilidade e compreensão do sistema e a satisfação do usuário. No que se refere ao uso e à utilidade do sistema AHP MAKH-ER, verifica-se de um modo geral, que a avaliação é boa, pois $62 \%$ das respostas encontram-se nas escalas 1 e 2 (concordo plenamente e concordo em grande parte), e nenhum caso extremamente negativo se destaca nesta avaliação. Chama a atenção o item "decisão mais confiável”, em que a maioria dos respondentes concorda em parte que o sistema propicia uma decisão de compra de carro mais confiável. 
Com relação à avaliação da interface do sistema, onde em uma avaliação geral, 78\% dos respondentes responderam 1 e 2 na escala, consideram-se positivos os aspectos da interface. Um item que chama a atenção é "não há dificuldade na interface", que apesar de no geral ter boa avaliação, as mesmas se distribuíram um pouco mais ao longo da escala, demonstrando que a concordância não é tão alta, e indicando que a visualização e manuseio na interface do sistema podem ser melhorados.

Também é possível observar na Tabela 1 que, para $85 \%$ dos respondentes o $A H P M A K H$-ER é fácil quanto ao seu uso e facilita compreensão, obtendo assim uma ótima avaliação referente à variável facilidade e compreensão do sistema.

Referente à avaliação geral da satisfação do usuário verifica-se que $91 \%$ deles assinalaram as opções $1 \mathrm{e}$ 2 na escala. Dessa forma, o AHP MAKH-ER pode ser validado com um Sistema de Apoio à Decisão, com uma boa avaliação perante seus usuários. Cabe destacar que esta boa avaliação pode se dar pelo fato de que $70 \%$ dos usuários informaram no início do questionário já terem escolhido um carro anteriormente, o que indica que conhecer o objeto de escolha influencia na avaliação do sistema que auxilia na escolha deste objeto.

Tabela 1 - Avaliação pelo usuário do sistema AHP MAKH-ER

\begin{tabular}{|c|c|c|c|c|c|}
\hline \multirow{2}{*}{ Item avaliado } & \multicolumn{5}{|c|}{ Escala } \\
\hline & 1 & 2 & 3 & 4 & 5 \\
\hline \multicolumn{6}{|c|}{ Avaliação do uso e da utilidade do sistema AHP MAKH-ER } \\
\hline Decisões mais rápidas e seguras & 6 & 10 & 6 & 0 & 0 \\
\hline Retrata a forma de raciocínio & 8 & 9 & 5 & 0 & 0 \\
\hline Decisão mais confiável & 3 & 7 & 11 & 1 & 0 \\
\hline Decisões mais fáceis & 5 & 8 & 8 & 1 & 0 \\
\hline Total & 22 & 34 & 32 & 2 & $\mathbf{0}$ \\
\hline \multicolumn{6}{|c|}{ Avaliação da interface do sistema AHP MAKH-ER } \\
\hline O layout permite fácil visualização e entendimento & 12 & 7 & 2 & 1 & 0 \\
\hline AHP MAKH-ER apresenta fácil acesso às variáveis & 9 & 6 & 5 & 1 & 1 \\
\hline O julgamento de critérios/carros é de fácil execução & 9 & 7 & 5 & 1 & 0 \\
\hline Os ícones facilitam o deslocamento & 10 & 8 & 3 & 1 & 0 \\
\hline Os gráficos apresentam as escolhas de forma clara & 12 & 7 & 2 & 0 & 1 \\
\hline Em geral, AHP MAKH-ER é de fácil utilização & 11 & 8 & 2 & 0 & 1 \\
\hline Não há dificuldade na interface & 8 & 6 & 6 & 2 & 0 \\
\hline Total & 71 & 49 & 25 & 6 & 3 \\
\hline \multicolumn{6}{|c|}{ Avaliação do sistema AHP MAKH-ER quanto à facilidade e compreensão } \\
\hline É fácil aprender a operar com o AHP MAKH-ER & 17 & 4 & 0 & 0 & 1 \\
\hline AHP MAKH-ER é fácil de usar & 16 & 5 & 0 & 0 & 1 \\
\hline AHP MAKH-ER é interativo & 14 & 6 & 2 & 0 & 0 \\
\hline A linguagem e o vocabulário são fáceis & 12 & 7 & 2 & 0 & 1 \\
\hline As informações sobre critérios e alternativas atendem sua necessidade & 10 & 8 & 3 & 0 & 1 \\
\hline O layout facilita a compreensão & 7 & 10 & 5 & 0 & 0 \\
\hline $\begin{array}{l}\text { Diferenciar modelos de carro numa escala de pouca importância a extrema } \\
\text { importância retrata a realidade }\end{array}$ & 7 & 8 & 6 & 1 & 0 \\
\hline Total & 83 & 48 & 18 & 1 & 4 \\
\hline \multicolumn{6}{|c|}{ Avaliação do sistema AHP MAKH-ER quanto à satisfação do usuário } \\
\hline O sistema AHP MAKH-ER atendeu suas expectativas & 12 & 9 & 0 & 1 & 0 \\
\hline AHP MAKH-ER auxilia sua decisão acerca de um carro & 7 & 13 & 1 & 1 & 0 \\
\hline Indica AHP MAKH-ER para alguém disposto a adquirir um carro & 13 & 6 & 1 & 1 & 1 \\
\hline Total & 32 & 28 & 2 & 3 & 1 \\
\hline
\end{tabular}


Ao final do questionário foi solicitado ao tomador de decisão, através de questão aberta, sua opinião sobre mudanças ou alterações no sistema. Dentre as observações: "o sistema está bem completo"; "os gráficos são bem representativos"; "o sistema realmente auxilia na decisão"; e "o sistema é extremamente válido". Aqui nota-se que o sistema é visto como capaz de auxiliar e explicar uma decisão.

\subsection{Validação do mapeamento do processo pelo sistema de apoio à decisão AHP MAKH-ER}

O sistema AHP MAKH-ER possibilita o estudo do processo decisório do usuário para chegar à decisão final. Todo esse processo é mapeado através de registro automático e da catalogação das ações tomadas pelo decisor para chegar a sua decisão. Os acessos computacionais (PAYNE; BETTMAN ; JOHNSON, 1993), chamadas de $\log s$, são mapeados pelo sistema e gerados em software Excel, como demonstrado na Figura 8, resultado de uma das etapas de validação pelo usuário.

O mapeamento permite verificar diferenças no modo de busca das informações contidas nas alternativas $\mathrm{X}$ critérios. Com os $\log s$ o pesquisador verifica o caminho que o decisor fez para chegar à decisão final, suas escolhas e julgamentos. Este mapeamento do processo ou traçado de decisão foi proposto por Payne, Bettman e Johnson, (1993) com o sistema Mouselab, em que propuseram um sistema que mapeava o processo de decisão, por meio do cursor do mouse, sendo mais eficaz do que acompanhar o movimento do olho humano.

O sistema AHP MAKH-ER, nesse sentido, tem a capacidade de monitorar o comportamento de obtenção da informação por parte do decisor, isto é, qual a informação acessada, qual a seqüência dessa aquisição, quantas vezes a mesma informação foi acessada, bem como o tempo de duração do exame de cada informação. A fim de validar o sistema no que tange a capacidade de mapeamento do processo, são apresentados a seguir os testes que verificam a possibilidade do sistema de mapear as variáveis citadas. Através de inputs controlados, verificou-se se estes foram retratados nos logs.

Na Figura 8 é possível identificar a ordem em que o indivíduo buscou as informações, se analisou por critério (todas as alternativas referentes a um critério), ou se buscou por alternativa (todos os critérios de uma alternativa). No exemplo, verifica-se que o decisor inicialmente não teve regra, mas que a partir do quarto clique (Marca), optou por examinar por critério, isto é, todas as informações dos três carros para o critério Marca. É possível estudar também o tempo total que o decisor leva para realizar a tarefa, bem como o tempo que permanece analisando uma informação, e o tempo que leva para decidir, ou seja, para escolher e ponderar as alternativas na tela pop up. Para essas verificações basta fazer a diferença entre o valor posterior (Resposta do pop up) e o anterior (Aberto pop up).

$\mathrm{O}$ arquivo dos $\log s$ permite ainda que se verifique a consistência dos julgamentos do usuário, isto é, se ele comete inconsistências durante seu processo decisório ou não (abordado na seção 3), bem como as transgressões cometidas por ele. Assim, sob pressão do tempo ou da falta de informação durante a tarefa decisória, as inconsistências e transgressões podem ser mensuradas como erros cometidos pelo decisor. $\mathrm{O}$ exemplo da Figura 8 mostra duas situações: uma de consistência, quando a Razão de Consistência (RC) é igual a 0,025183; e outra de inconsistência quando $\mathrm{RC}=0,103483$.

\begin{tabular}{|c|c|c|c|c|}
\hline 4 & $\mathrm{~A}$ & $\mathrm{~B}$ & $\mathrm{C}$ & D \\
\hline 1 & Criterio & Objeto & Data & Hora \\
\hline 2 & Clicou em: PREÇO & GOL CITY & $10 / 01 / 2009$ & $14: 38: 38: 40$ \\
\hline 3 & Clicou em: PREÇO & CELTA LIFE & $10 / 01 / 2009$ & $14: 38: 40: 01$ \\
\hline 4 & Clicou em: CONSUMO - Gas. & GOL CITY & $10 / 01 / 2009$ & $14: 38: 41: 72$ \\
\hline 5 & Clicou em: MARCA & GOL CITY & $10 / 01 / 2009$ & $14: 38: 46: 37$ \\
\hline 6 & Clicou em: MARCA & CELTA LIFE & $10 / 01 / 2009$ & $14: 38: 47: 89$ \\
\hline 7 & Clicou em: MARCA & PALIO FIRE & $10 / 01 / 2009$ & $14: 38: 49: 04$ \\
\hline 8 & Aberto popup MARCA: PALIO FIREXGOL CITY & & $10 / 01 / 2009$ & $14: 38: 50: 09$ \\
\hline 9 & Resposta do pop up: GOL CITY - intensidade $=9$ & & $10 / 01 / 2009$ & $14: 38: 56: 31$ \\
\hline 10 & Aberto popup MARCA: GOL CITYXCELTA LIFE & & $10 / 01 / 2009$ & $14: 38: 56: 46$ \\
\hline 11 & Resposta do pop up: GOL CITY - intensidade $=5$ & & $10 / 01 / 2009$ & 14:39:07:65 \\
\hline 12 & Aberto popup MARCA: CELTA LIFEXPALIO FIRE & & $10 / 01 / 2009$ & 14:39:07:84 \\
\hline 13 & Resposta do pop up: CELTA LIFE - intensidade $=5$ & & $10 / 01 / 2009$ & $14: 39: 12: 66$ \\
\hline 14 & $\mathrm{RC}=0.103483$ & & $10 / 01 / 2009$ & $14: 39: 12: 85$ \\
\hline 15 & Clicou em: CONSUMO - Gas. & CELTA LIFE & $10 / 01 / 2009$ & $14: 39: 49: 75$ \\
\hline 16 & Clicou em: CONSUMO - Gas. & PALIO FIRE & $10 / 01 / 2009$ & $14: 39: 50: 29$ \\
\hline 17 & Aberto popup CONSUMO: PALIO FIREXGOL CITY & & $10 / 01 / 2009$ & $14: 39: 51: 36$ \\
\hline 18 & Resposta do pop up: PALIO FIRE - intensidade $=5$ & & $10 / 01 / 2009$ & $14: 39: 54: 03$ \\
\hline 19 & Aberto popup CONSUMO: GOL CITYXCELTA LIFE & & $10 / 01 / 2009$ & $14: 39: 54: 17$ \\
\hline 20 & Resposta do pop up: CELTA LIFE - intensidade $=9$ & & $10 / 01 / 2009$ & $14: 39: 55: 91$ \\
\hline 21 & Aberto popup CONSUMO: CELTA LIFEXPALIO FIRE & & $10 / 01 / 2009$ & $14: 39: 56: 07$ \\
\hline 22 & Resposta do pop up: CELTA LIFE - intensidade $=3$ & & $10 / 01 / 2009$ & $14: 39: 57: 92$ \\
\hline 23 & $\mathrm{RC}=0.025183$ & & $10 / 01 / 2009$ & 14:39:58:08 \\
\hline
\end{tabular}

Figura 8 - Arquivo log.xls gerado pelo AHP MAKH-ER - Tarefa com o mapeamento das ações do decisor, seus julgamentos e razão de consistência. 
Já as transgressões são verificadas por meio das respostas das telas pop up, ou seja, das intensidades julgadas pelo decisor. A Figura 8 mostra os julgamentos das intensidades entre as linhas 8 a 13, bem como linhas 17 a 22 da planilha. Conforme a Teoria da Imagem, o indivíduo após definir mentalmente um determinado estado final como desejável, passa a transgredir regras de coesão e transitividade para que a decisão seja aquela previamente decidida (LÖBLER, 2006). Uma transgressão seria o exemplo que segue. Dados os valores para consumo de combustível em $\mathrm{km} / \mathrm{l}$, dos carros A, B e C respectivamente: $15,17,12$, então 17>15>12. Supõe-se a hierarquização dos valores nessa ordem, caso contrário não é possível determinar uma relação de dominância. Se o decisor atribuir que 17>12>15, obtém-se uma transgressão, pois estará afirmando que o consumo do carro C (12 km/l) é maior/melhor que o do carro A (15 km/l). Neste exemplo, o indivíduo por previamente desejar o carro $\mathrm{C}$, comete transgressões durante a tarefa decisória.

A utilização do sistema $A H P M A K H$-ER permitiu validar o mapeamento do processo. O mapeamento explica o processo como um todo, sobretudo se o pesquisador tiver interesse em verificar diferenças no processo decisório de grupos com características diferentes, ou submetidos a uma variável independente, como a pressão do tempo e a falta de informação durante a realização da tarefa.

\section{Considerações finais}

Ao final desse trabalho, espera-se ter cumprido com o objetivo proposto. Por meio do processo de validação descrito, baseado na avaliação pelo usuário bem como na validação de mapeamento do processo, acredita-se que o AHP MAKH-ER pode ser utilizado como ferramenta de apoio para se estudar a pressão do tempo e a falta de informação no processo decisório, identificando diferentes comportamentos elencados pela literatura.

A avaliação dos usuários foi de que o AHP MAKH-ER é um sistema válido, que realmente auxilia na decisão. Dessa forma, se valida também o método multicritério AHP, o qual sustenta o referido sistema, e que além da quantificação dos resultados, por meio das inconsistências e transgressões (erros) cometidas pelo decisor, busca apoiar o estudo da compreensão da mente humana quando em diferentes situações, auxiliando o pesquisador a entender o comportamento dos indivíduos no processo decisório.

O AHP MAKH-ER é um Sistema de Apoio à Decisão totalmente flexível, devido ao seu módulo $A H P$ $M A K H-E R$ - Gerenciador, podendo ser adaptado a várias situações de tomada de decisão, isto é, gerando diferentes tarefas decisórias, com a quantidade de critérios e alternativas desejadas pelo pesquisador. Assim, possibilita que experimentos sejam realizados não só por meio da tarefa decisória apresentada neste estudo, mas qualquer outro tipo de tarefa que simule uma escolha ou uma decisão.

Para pesquisas futuras, sugere-se que o AHP MAKH-ER seja aplicado, em forma de experimento, sob diferentes combinações das variáveis pressão do tempo e falta de informação permitindo aprofundar os estudos sobre o comportamento do decisor.

\section{Referências}

AHITUV, N.; IGBARIA, M.; SELLA, A. The Effects of Time Pressure and Completeness of Information on Decision Making. Journal of Management Information Systems, v.15, n. 2, p. 153-172, 1998.

BETENCOURT, P. R. B.; BORENSTEIN, D. Desenvolvimento de um Modelo de Decisão com Múltiplos Critérios para a Justificativa de Investimentos em TI. In: ENCONTRO DA ANPAD, 26., Salvador, 2002. Anais..., Rio de Janeiro: Associação Nacional de Pós-Graduação em Administração, 2002.

BIASIO, R. Concepção, desenvolvimento e validação de um sistema de apoio à decisão comercial - SADEC. 2002. Dissertação (Mestrado em Administração) - Escola de Administração, Programa de Pós-Graduação em Administração, Universidade Federal do Rio Grande do Sul, Porto Alegre, 2002.

BIASIO, R.; BORENSTEIN, D. Um Estudo sobre a Influência do Ambiente e do Avaliador na Validação de um Sistema de Apoio à Decisão-SAD. In: ENCONTRO DA ANPAD, 30., Salvador, 2006. Anais..., Rio de Janeiro: Associação Nacional de Pós-Graduação em Administração, 2006.

BORENSTEIN, D. Ranking: um sistema de apoio a decisões multicriteriais. Revista de Administração. São Paulo, v.32, n.4, p. 67-76, Out./Dez. 1997.

BORENSTEIN, D. Toward a method to validate decision support systems. Decision Support Systems, v. 23, p. 227-239, 1998.

BORENSTEIN, D.; BECKER, J. L. Validating Decision Support Systems. Encyclopedia of Microcomputers. New York, v.26, n.5, p. 323-341, 2000.

BURREL, G.; MORGAN, G. Sociological paradigms and organizational analysis. London: Heinemann, 1979. 
FISHER, C. W.; CHENGALUR-SMITH, I. S.; BALLOU, D. P. The impact of experience and time on the use of data quality information in decision making. Information Systems Research, v.14, n.2, p. 170-188, Jun. 2003.

FREZATTI, F. et al. Análise do Relacionamento entre a Contabilidade Gerencial e o Processo de Planejamento das Organizações Brasileiras. In: ENCONTRO DA ANPAD, 29., Brasília, 2005. Anais..., Rio de Janeiro: Associação Nacional de Pós-Graduação em Administração, 2005.

GALVÃO, A. M.; COGAN, S.; SANTOS, G. P. dos. Um Modelo Simplificado de Custeio a partir do ABC/ AHP: Estudo e Análise em uma Empresa de Turismo. In: ENCONTRO DA ANPAD, 26., Salvador, 2002. Anais..., Rio de Janeiro: Associação Nacional de PósGraduação em Administração, 2002.

GOMES, L. F. A. M.; ARAYA, M. C. G.; CARIGNANO, C. Tomada de Decisões em Cenários Complexos. São Paulo: Pioneira Thomson Learning, 2004. 168p.

HAHN, M.; LAWSON, R.; LEE, Y. G. The Effect of Time Pressure and Information Load on Decision Quality. Psychology e Marketing, v.9, n.5, p. 365-378, 1992.

HOPPEN, N.; LAPOINTE, L.; MOREAU, E. Um guia para avaliação de artigos de pesquisa em sistemas de Informação. Série documentos para estudo. PPGA/ UFGRS, nº 09/96, 1996.

IAÑEZ, M. M.; CUNHA, C. B. Uma metodologia para a seleção de um provedor de serviços logísticos. Produção, v. 16, n. 3, p. 394-412, Set./Dez. 2006.

JAGACINSKI, C. M. Personnel Decision Making: The Impact of Missing Information. Journal of Applied Psychology, v.76, n.1, p. 19-30, 1991.

JIMÉNEZ, A.; MATEOS, A.; RÍOS-INSUA, S. Missing consequences in multiattribute utility theory. Omega, v.37, p. 395-410, 2009.

KAHNEMAN, D.; TVERSKY, A. Choice, Values and Frames. Cambridge, Universtity Press, Cambridge, 2000. 860p.

KIVETZ, R.; SIMONSON, I. The Effects of Incomplete Information on Consumer Choice. Journal of Marketing Research, v.37, p. 427-448, 2000.

KÖRNER, C.; GERTZEN, H.; BETTINGER, C.; ALBERT, D. Comparative judgments with missing information: A regression and process tracing analysis. Acta Psychologica, v.125, p. 66-84, 2007.
LIBERATORE, M. J.; NYDICK, R. L. The analytic hierarchy process in medical and health care decision making: A literature review. European Journal of Operational Research, v.189, p. 194-207, 2008.

LÖBLER, M. L.; HOPPEN, N. Uso da Informação e Estratégias de Decisão na Interação com um SAD. In: ENCONTRO DA ANPAD, 29., Brasília, 2005. Anais..., Rio de Janeiro: Associação Nacional de Pós-Graduação em Administração, 2005.

LÖBLER, M. L.; HOPPEN, N. Validação de Decisor, um sistema de apoio à decisão multicriterial para mapear processos decisórios. Revista Eletrônica de Administração - REAd, Edição 49, v.12, n. 1, Jan./Fev. 2006.

LÖBLER, M. L. A Teoria da Imagem como Explicação para Violação do Método Multicritério de Decisão. In: ENCONTRO DA ANPAD, 30., Salvador, 2006. Anais..., Rio de Janeiro: Associação Nacional de Pós-Graduação em Administração, 2006.

MATOS, D. M.; MOURA, H. J. Proposta de um Modelo para Determinação do Custo de Capital Baseado em Análise Hierárquica. Revista de Administração Contemporânea - RAC, v.7, n.4, p. 119-139, Out./Dez. 2003.

ORDÓÑEZ, L.; BENSON III, L.; Decisions under Time Pressure: How Time Constraint Affects Risky Decison Making. Organizational Behavior and Human Decision Processes, v.71, n.2, p. 121-140, Aug. 1997.

PAYNE, J. W.; BETTMAN, J. R.; JOHNSON, E. J. The adaptive decision maker. Cambridge University Press. Cambridge, 1993. 330p.

PAYNE, J. W.; BETTMAN, J. R.; LUCE, M. F. When Time Is Money: Decision Behavior under Opportunity-Cost Time Pressure. Organizational Behavior and Human Decision Processes, v.66, n.2, p. 131-152, 1996.

QUATRO RODAS. Revista Quatro Rodas. Testes. São Paulo, 2008. Disponível em: <http://quatrorodas.abril. com.br/QR2/carros/testes/>. Acesso em: 14 nov. 2008.

RAFAELI, L.; MÜLLER, C. J. Estruturação de um índice consolidado de desempenho utilizando o AHP. Gestão \& Produção, v.14, n.2, p. 363-377, Mai./Ago. 2007.

SANGLARD JUNIOR, M. R.; GOMES, L. F. A. M. Uma Aplicação Prática da Análise Multicritério na Decisão de Desenvolvimento de Software com Equipe Interna ou Terceirizada: Estudo de Caso em Uma Empresa de Telecomunicações. In: ENCONTRO DA ANPAD, 27., Atibaia, 2003. Anais..., Rio de Janeiro: Associação Nacional de Pós-Graduação em Administração, 2003. 
SAATY, T. L. Método de análise hierárquica. São Paulo: Makron Books, 1991. 367p.

SHIMIZU, T. Decisão nas organizações. 2 ed. São Paulo: Atlas, 2006. 317p.

SIMONETTO, E. O.; BORENSTEIN, D. Validação de um sistema de apoio à decisão para o planejamento da coleta seletiva de resíduos. Anais do XXVIII Encontro Nacional de Engenharia de Produção, 2008.

SMITH, C. A. P.; HAYNE, S. C. Decision making under time pressure: An Investigation of Decision Speed and Decision Quality of Computer-Supported Groups. Management Communication Quarterly, v. 11, p. 97126, 1997. 